\title{
Isotope Shifts in High Lying Levels of Dy I and Er I by High-Resolution UV Laser Spectroscopy
}

\author{
Wei-Guo Jin, Hiroaki Ono, and Tatsuya Minowa \\ Department of Physics, Faculty of Science, Toho University, Miyama 2-2-1, Funabashi, Chiba 274-8510, Japan \\ Correspondence should be addressed to Wei-Guo Jin, jin@ph.sci.toho-u.ac.jp
}

Received 15 September 2011; Accepted 6 October 2011

Academic Editor: Kam-Sing Wong

Copyright () 2011 Wei-Guo Jin et al. This is an open access article distributed under the Creative Commons Attribution License, which permits unrestricted use, distribution, and reproduction in any medium, provided the original work is properly cited.

High-resolution atomic-beam ultraviolet (UV) laser spectroscopy in Dy I and Er I has been performed. Isotope shifts have been measured for two transitions in Dy I and one transition in Er I. Specific mass shifts and field shifts have been derived for the studied transitions, and large differences between the two $4 f^{10} 6 s^{2}-4 f^{10} 6 s 6 p$ transitions in Dy I have been found. From the derived specific mass shifts and field shifts, configuration mixing at the upper levels of transitions has been discussed.

\section{Introduction}

High-resolution laser spectroscopy continues to play an important role in obtaining atomic spectroscopic data such as isotope shift (IS). The IS contains fundamental atomic properties such as electronic configuration and wave function. Recently, theoretical calculations of IS have been reported by different groups using various methods for light elements of Li, Na, and Mg [1-3]. Dysprosium and erbium, two typical rare-earth elements, have the $4 f, 5 d, 6 s, 6 p$ open shells, which yield complicated atomic structures. Such a kind of complex heavy atoms provides a challenge to theoretical atomic calculations of many-electron atoms [4]. Moreover, Dy and Er are prime candidates for the study of ultracold dipolar physics $[5,6]$. On the other hand, measurements of IS yield the mass dependence of nuclear charge radii, a key information for the study of unstable nuclei $[7,8]$. Study of IS is, therefore, of much interest not only from the point of view of atomic physics but also from the point of view of nuclear physics.

Many studies of IS have been reported for Dy I and Er I in the visible and near-infrared regions by laser spectroscopy [5, 9-16]. For the ultraviolet (UV) region, corresponding to high lying levels at energy about $25000 \mathrm{~cm}^{-1}$, only several measurements have been reported. ISs in high lying levels of Dy I have been measured by using a Fabry-Perot spectrometer [17] and those of Er I by Doppler-reduced saturation absorption spectroscopy [18]. Strong configuration mixing is considered in such high lying levels and may yield different ISs.

In our previous papers [19-21], we reported high-resolution atomic-beam UV laser spectroscopy in Gd I and Er I around $395 \mathrm{~nm}$ by frequency doubling of a diode laser beam. The present work is to extend previous measurements to the wavelength region of about $402 \mathrm{~nm}$ in Dy I and Er I. In this paper, ISs are measured for two UV transitions in Dy I and one transition in Er I. From ISs, field shifts and specific mass shifts are obtained, and results are discussed.

\section{Experiment}

The present experiment was performed using an atomic beam and a UV laser beam. The experimental setup is essentially identical to that used in our previous experiments [1921]. An atomic vapor was ejected from a 1-mm-diameter hole of a molybdenum oven heated to about $1400^{\circ} \mathrm{C}$ by an electron-bombardment heating method. An atomic beam was formed by a 2-mm-diameter aperture at a distance of $30 \mathrm{~cm}$ from the oven.

A laser beam with a wavelength of about $804 \mathrm{~nm}$ and a power of about $20 \mathrm{~mW}$ was produced using a commercial tunable diode laser with an external cavity system (Newport 2010M). A UV beam with a wavelength of about $402 \mathrm{~nm}$ and a power of about $10 \mu \mathrm{W}$ was obtained by frequency 
TABLE 1: Wavelengths of the studied transitions and properties of the lower and upper levels in Dy I and Er I.

\begin{tabular}{lccccccc}
\hline \multirow{2}{*}{ Element } & Wavelength $(\mathrm{nm})$ & & Lower level & \multicolumn{3}{c}{ Upper level } \\
& & Configuration & Level & Energy $\left(\mathrm{cm}^{-1}\right)$ & Configuration & $\begin{array}{c}\text { Level } \\
\text { Energy }\left(\mathrm{cm}^{-1}\right)\end{array}$ \\
\hline Dy & 401.382 & $4 f^{10} 6 s^{2}$ & ${ }^{5} I_{8}$ & 0.0 & $4 f^{10} 6 s 6 p$ & $(6,2)_{7}$ & 24906.86 \\
& 404.597 & $4 f^{10} 6 s^{2}$ & ${ }^{5} I_{8}$ & 0.0 & $4 f^{10} 6 s 6 p$ & $(8,1)_{7}$ & 24708.96 \\
Er & 400.796 & $4 f^{12} 6 s^{2}$ & ${ }^{3} H_{6}$ & 0.0 & $4 f^{12} 6 s 6 p$ & $(6,1)_{7}$ & 24943.272 \\
\hline
\end{tabular}

TABLE 2: Measured isotope shifts in Dy I and Er I.

\begin{tabular}{|c|c|c|c|c|c|}
\hline \multirow{2}{*}{$\begin{array}{l}\text { Element } \\
\text { Dy }\end{array}$} & \multirow[t]{2}{*}{ Wavelength $(\mathrm{nm})$} & \multicolumn{4}{|c|}{ Isotope shift $(\mathrm{MHz})$} \\
\hline & & $164-162$ & $162-160$ & $160-158$ & $158-156$ \\
\hline & 401.382 & $-959.0(16)$ & $-1036.5(18)$ & $-1023.1(28)$ & $-1520.4(36)$ \\
\hline & 404.597 & $-375.7(14)$ & $-398.3(15)$ & $-397.9(23)$ & $-530.2(29)$ \\
\hline \multirow[t]{2}{*}{ Er } & & $170-168$ & $168-166$ & $166-164$ & $164-162$ \\
\hline & 400.796 & $-866.5(15)$ & $-855.2(17)$ & $-859.3(16)$ & $-1028.7(28)$ \\
\hline
\end{tabular}

doubling using a cw frequency doubler (Spectra-Physics, Wavetrain).

The laser beam crossed the atomic beam perpendicularly in order to reduce the Doppler broadening. A spherical mirror was used to collect laser-induced fluorescence from the atomic beam which was detected with a cooled photoncounting photomultiplier (Hamamatsu R2257P). An interference filter with a $40 \mathrm{~nm}$ width at $400 \mathrm{~nm}$ was used in front of the photomultiplier, and, therefore, the background from the intense oven light was greatly suppressed. The spectrum of transmitted light through a confocal Fabry-Perot interferometer (FPI) with a free spectral range of $300 \mathrm{MHz}$ was measured simultaneously with the fluorescence spectrum for relative frequency calibration.

\section{Results}

Two transitions in Dy I and one transition in Er I were studied in this experiment. Wavelengths of the transitions, electronic configurations, and energies of the lower and upper levels [22-24] are presented in Table 1. For Dy, two transitions are from the ground state $4 f^{10} 6 s^{25} I_{8}$ to the levels of the $4 f^{10} 6 s 6 p$ configuration. For Er, the transition is from the ground state $4 f^{12} 6 s^{2}{ }^{3} H_{6}$ to the $(6,1)_{7}$ level of the $4 f^{12} 6 s 6 p$ configuration. All transitions belong to $s^{2}-s p$ transitions.

Figure 1 shows the observed fluorescence spectrum for the $400.796 \mathrm{~nm}$ transition in Er I. Peaks of the four even-mass isotopes ${ }^{170} \mathrm{Er},{ }^{168} \mathrm{Er},{ }^{166} \mathrm{Er},{ }^{164} \mathrm{Er}$ could be easily identified from their peak intensities together with their isotopic abundances. The peak of the lowest abundance $(0.14 \%)$ isotope ${ }^{162} \mathrm{Er}$ was also clearly observed. Other peaks are hyperfine structure peaks of the odd-mass isotope ${ }^{167} \mathrm{Er}$. ${ }^{167} \mathrm{Er}$, with the nuclear spin of $7 / 2$, has rich hyperfine structure peaks which are mainly overlapped with strong peaks of even-mass isotopes. The full width at half maximum (FWHM) of peaks is approximately $70 \mathrm{MHz}$ and is mainly from the natural width of the upper level of the transition and the residual Doppler effect of the interaction between the atomic beam and laser beam; the linewidth of the laser is less than $1 \mathrm{MHz}$.

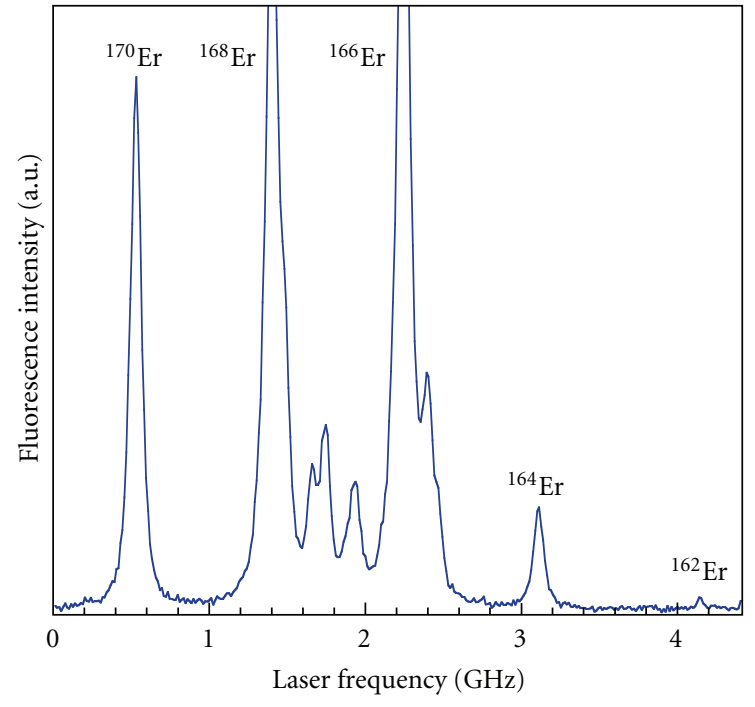

Figure 1: Observed fluorescence spectrum of the $4 f^{12} 6 s^{2}{ }^{3} H_{6}-$ $-4 f^{12} 6 s 6 p(6,1)_{7}$ transition at $400.796 \mathrm{~nm}$ in Er I. Peaks of the evenmass isotopes are labeled with their atomic symbol. Other peaks are hyperfine structure peaks of the odd-mass isotope ${ }^{167} \mathrm{Er}$.

Measured spectra were fitted with a Lorentz function and calibrated with the FPI spectra. For each transition, measurement was repeated about 20 times. Thus, ISs were obtained for the studied transitions and are listed in Table 2. The uncertainties of measured ISs are $2-4 \mathrm{MHz}$ and contain the error of peak-center determination, the error of the free spectral range of the FPI $(0.046 \mathrm{MHz})$, and the error of linearity correction for frequency scanning.

For Dy, the IS is $-959 \mathrm{MHz}$ for the isotope pair of 164162 for the $401.382 \mathrm{~nm}$ transition while the IS is $-376 \mathrm{MHz}$, more than twice smaller, for the $404.597 \mathrm{~nm}$ transition. For the Er $400.796 \mathrm{~nm}$ transition, the IS is $-867 \mathrm{MHz}$ for the isotope pair of $170-168$. 
TABLE 3: Determined SMS factors $M_{i S M S}$ and electronic factors $F_{i}$ in Dy I and Er I.

\begin{tabular}{lccc}
\hline Element & Wavelength $(\mathrm{nm})$ & $M_{i \mathrm{SMS}}\left(10^{5} \mathrm{MHz}\right)$ & $F_{i}\left(\mathrm{GHz} / \mathrm{fm}^{2}\right)$ \\
\hline Dy & 401.382 & $-1.5(30)$ & $-8.21(45)$ \\
& 404.597 & $-20.4(16)$ & $-2.12(13)$ \\
Er & 400.796 & $-13.4(62)$ & $-7.86(53)$ \\
\hline
\end{tabular}

TABle 4: Derived specific mass shifts and field shifts in Dy I and Er I.

\begin{tabular}{lccccccccc}
\hline Element & Wavelength $(\mathrm{nm})$ & \multicolumn{3}{c}{ Specific mass shift $(\mathrm{MHz})$} & \multicolumn{4}{c}{ Field shift $(\mathrm{MHz})$} \\
\hline Dy & & $164-162$ & $162-160$ & $160-158$ & $158-156$ & $164-162$ & $162-160$ & $160-158$ & $158-156$ \\
& 401.382 & $-11(22)$ & $-11(23)$ & $-12(24)$ & $-12(24)$ & $-979(22)$ & $-1057(23)$ & $-1044(24)$ & $-1542(24)$ \\
& 404.597 & $-153(12)$ & $-157(12)$ & $-161(13)$ & $-165(13)$ & $-253(12)$ & $-272(13)$ & $-269(13)$ & $-398(13)$ \\
Er & & $170-168$ & $168-166$ & $166-164$ & $164-162$ & $170-168$ & $168-166$ & $166-164$ & $164-162$ \\
& 400.796 & $-94(44)$ & $-96(45)$ & $-98(46)$ & $-101(47)$ & $-801(44)$ & $-788(45)$ & $-791(46)$ & $-959(47)$ \\
\hline
\end{tabular}

\section{Analysis and Discussion}

The isotope shift $\delta v_{i}$ between the isotopes with the mass numbers $A$ and $A^{\prime}$, for a transition $i$, contains the mass shift and field shift (FS). The mass shift includes the normal mass shift (NMS) and specific mass shift (SMS) $[25,26]$. The NMS $\delta \nu_{i \mathrm{NMS}}$, due to the change in the reduced mass of electron and nucleus, can be calculated easily using the transition frequency. The SMS $\delta v_{i S M S}$ is caused by the influence of correlations in the motion of electrons on the recoil energy of the nucleus and is expressed as

$$
\delta v_{i S M S}=M_{i S M S} \frac{A^{\prime}-A}{A A^{\prime}},
$$

where $M_{i S M S}$ is the factor of the SMS. The FS $\delta \nu_{i F S}$ originates from the change in the nuclear charge distribution and is written as

$$
\delta \nu_{i \mathrm{ES}}=F_{i} \lambda,
$$

where $F_{i}$ is the electronic factor related to the change of the electron charge density at the nucleus and $\lambda$ is called the nuclear parameter associated with the change in meansquare nuclear charge radii $\delta\left\langle r^{2}\right\rangle$ [26].

When the modified IS $\delta v_{i}^{\text {mod }}$ and modified nuclear parameter $\lambda^{\text {mod }}$ are defined as

$$
\begin{gathered}
\delta v_{i}^{\mathrm{mod}}=\left(\delta v_{i}-\delta v_{i \mathrm{NMS}}\right) \frac{A A^{\prime}}{A^{\prime}-A} \cdot \frac{2}{A_{1} \cdot A_{2}}, \\
\lambda^{\mathrm{mod}}=\lambda \frac{A A^{\prime}}{A^{\prime}-A} \cdot \frac{2}{A_{1} \cdot A_{2}},
\end{gathered}
$$

a following linear relation is obtained:

$$
\delta v_{i}^{\bmod }=F_{i} \lambda^{\bmod }+M_{i S \mathrm{MS}} \frac{2}{A_{1} \cdot A_{2}},
$$

where $A_{1}=164, A_{2}=162$ for Dy and $A_{1}=170, A_{2}=168$ for Er. The relation of (4) is called King plot [27]. Using the knowing nuclear parameter $\lambda$, the SMS factor $M_{i S M S}$, and electronic factor $F_{i}$, that is, the SMS and FS, can be obtained from the measured IS by the King plot.

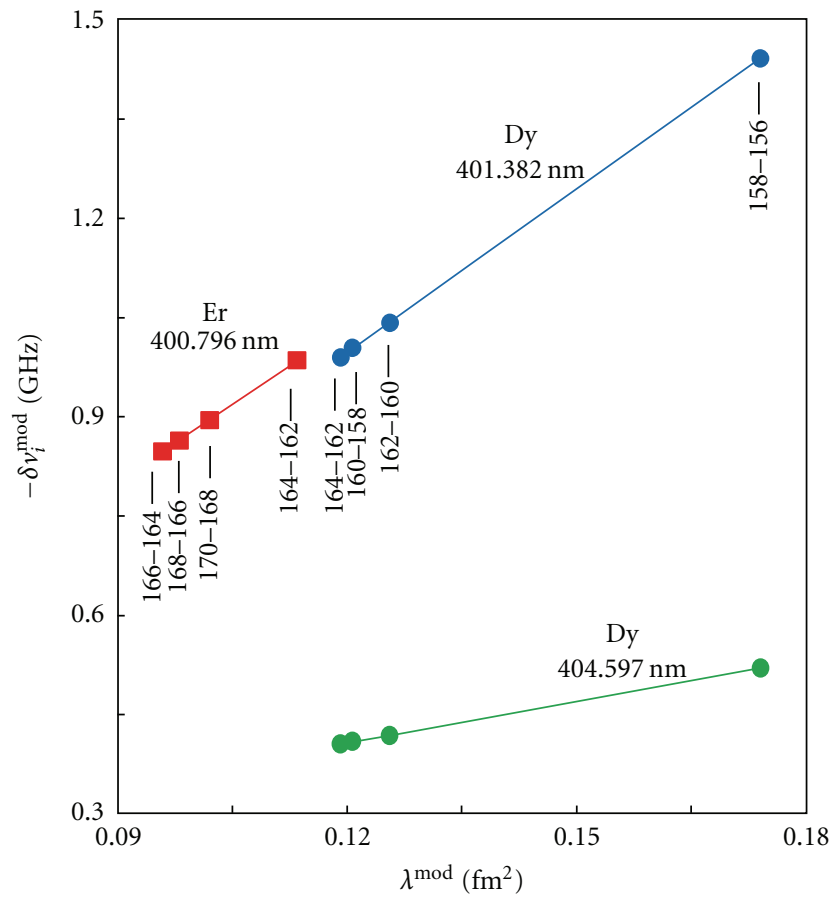

FIGURE 2: King plots for the three transitions in Dy I and Er I. Errors of both $\delta \nu_{i}^{\bmod }$, the $y$ axis, and $\lambda^{\text {mod }}$, the $x$ axis, are within symbols. Lines are the results of a least-squares fit.

Figure 2 shows King plots for the studied three transitions in Dy I and Er I. In the King plot we used values of the nuclear parameter $\lambda$ derived from optical IS measurements with the experimental uncertainty $[13,14]$. Good linear relations are seen for all King plots. From the King plot, the electronic factor $F_{i}$ and SMS factor $M_{i S M S}$ were obtained and are listed in Table 3 for all transitions studied. Uncertainty of 5\% was included in the error of the electronic factor $F_{i}$ as in [14]. Using the SMS factor $M_{i S M S}$, SMS was obtained and FS was further deduced from the measured IS. The derived SMSs and FSs are listed in Table 4 for the different isotope pairs. 
It can be seen from Table 4 that, for the Dy $401.382 \mathrm{~nm}$ transition, the SMSs are negligibly small within experimental uncertainties and the FSs, that is, the ISs, are comparable to those observed in the visible region [14]. This shows that the Dy $401.382 \mathrm{~nm}$ transition is a pure $s^{2}-s p$ transition. For the Dy $404.597 \mathrm{~nm}$ transition, however, the SMS is $-153 \mathrm{MHz}$ and the FS is $-253 \mathrm{MHz}$ for the isotope pair of 164-162, largely deviating from those of the $401.382 \mathrm{~nm}$ transition. This is considered to be due to strong configuration mixing at the upper level of the $404.597 \mathrm{~nm}$ transition. For the Er $400.796 \mathrm{~nm}$ transition, the SMS is $-94 \mathrm{MHz}$ and the FS is $-801 \mathrm{MHz}$ for the isotope pair of $170-168$, significantly deviating from those observed in the visible region [13]. This shows that significant configuration mixing exists at the upper level of the $400.796 \mathrm{~nm}$ transition in Er I.

For the odd-parity levels of Dy I and Er I, configurations of $4 f^{N} 6 s 6 p, 4 f^{N-1} 5 d 6 s^{2}$, and $4 f^{N-1} 5 d^{2} 6 s$ are involved and mixing between these three configurations is possible. For the transitions of $4 f^{10} 6 s^{2}-4 f^{9} 5 d 6 s^{2}$ in Dy I and $4 f^{12} 6 s^{2}-$ $4 f^{11} 5 d 6 s^{2}, 4 f^{12} 6 s^{2}-4 f^{11} 5 d^{2} 6 s$ in Er I, the SMS factor $M_{i S M S}$ and electronic factor $F_{i}$ have been reported and have a sane order of magnitude for Dy and $\operatorname{Er}[10,21]$. Using the derived SMS factor $M_{i S M S}$ and electronic factor $F_{i}$ in Table 3 and those reported for the transitions of $4 f^{10} 6 s^{2}-4 f^{9} 5 d 6 s^{2}$ in Dy I and $4 f^{12} 6 s^{2}-4 f^{11} 5 d 6 s^{2}, 4 f^{12} 6 s^{2}-4 f^{11} 5 d^{2} 6 s$ in $\mathrm{Er}$ I, percentage of configuration mixing at the upper levels of the studied transitions can be estimated. For the upper level $4 f^{10} 6 s 6 p(8,1)_{7}$ of the $404.597 \mathrm{~nm}$ transition in Dy I, considering a mixing from the $4 f^{9} 5 d 6 s^{2}$ configuration, we obtained 25(5)\% mixing using the SMS factor $M_{i S M S}$ and $27(4) \%$ mixing using the electronic factor $F_{i}$. However, by supposing a mixing from the $4 f^{9} 5 d^{2} 6 s$ configuration, mixing of $94(16) \%$ was estimated and was unreasonably too large. Therefore, we concluded that there is $26(3) \%$ mixing from the $4 f^{9} 5 d 6 s^{2}$ configuration at the upper level $4 f^{10} 6 s 6 p(8,1)_{7}$ of the $404.597 \mathrm{~nm}$ transition in Dy I. In a similar way, 10(3)\% mixing from the $4 f^{11} 5 d 6 s^{2}$ configuration at the upper level $4 f^{12} 6 s 6 p(6,1)_{7}$ of the $400.796 \mathrm{~nm}$ transition in Er I was estimated using the electronic factor $F_{i}$.

\section{Summary}

High-resolution laser spectroscopy in the UV region of Dy I and Er I has been performed using frequency doubling of the diode-laser beam together with the collimated atomic beam. ISs have been measured for two UV transitions in Dy I and one transition in Er I. SMSs and FSs as well as SMS factors $M_{i \text { SMS }}$ and electronic factors $F_{i}$ have been derived from the measured ISs using the known nuclear parameter. From the derived SMSs and FSs, it has been found that the $401.382 \mathrm{~nm}$ transition in Dy I can be considered as a pure $s^{2}-s p$ transition, and, for the upper level $4 f^{10} 6 s 6 p(8,1)_{7}$ of the $404.597 \mathrm{~nm}$ transition, strong mixing of $26(3) \%$ from the $4 f^{9} 5 d 6 s^{2}$ configuration was estimated. For the upper level $4 f^{12} 6 s 6 p(6,1)_{7}$ of the $400.796 \mathrm{~nm}$ transition in Er I, significant mixing from the $4 f^{11} 5 d 6 s^{2}$ configuration was found and estimated to be $10(3) \%$.

\section{Acknowledgment}

The authors thank Mr. S. Sakuma and Mr. T. Kurosaki for their help in this experiment.

\section{References}

[1] Z.-C. Yan, W. Nörtershäuser, and G. W. F. Drake, "High precision atomic theory for $\mathrm{Li}$ and $\mathrm{Be}^{+}: \mathrm{QED}$ shifts and isotope shifts," Physical Review Letters, vol. 100, no. 24, Article ID 243002, 2008.

[2] J. C. Berengut, V. V. Flambaum, and M. G. Kozlov, "Calculation of relativistic and isotope shifts in Mg I," Physical Review A, vol. 72, no. 4, Article ID 044501, 4 pages, 2005.

[3] M. S. Safronova and W. R. Johnson, "Third-order isotope-shift constants for alkali-metal atoms and ions," Physical Review A, vol. 64, no. 5, Article ID 052501, 12 pages, 2001.

[4] V. A. Dzuba and V. V. Flambaum, "Theoretical study of some experimentally relevant states of dysprosium," Physical Review A, vol. 81, no. 5, Article ID 052515, 2010.

[5] M. Lu, S. H. Youn, and B. L. Lev, "Spectroscopy of a narrowline laser-cooling transition in atomic dysprosium," Physical Review A, vol. 83, no. 1, Article ID 012510, 2011.

[6] A. J. Berglund, J. L. Hanssen, and J. J. McClelland, "Narrowline magneto-optical cooling and trapping of strongly magnetic atoms," Physical Review Letters, vol. 100, no. 11, Article ID 113002, 2008.

[7] H. J. Kluge and W. Nörtershäuser, "Lasers for nuclear physics," Spectrochimica Acta - Part B Atomic Spectroscopy, vol. 58, no. 6, pp. 1031-1045, 2003.

[8] W. Nörtershäuser, R. Sánchez, G. Ewald et al., "Isotope-shift measurements of stable and short-lived lithium isotopes for nuclear-charge-radii determination," Physical Review A, vol. 83, no. 1, Article ID 012516, 2011.

[9] N. Leefer, A. Cingöz, and D. Budker, "Measurement of hyperfine structure and isotope shifts in the Dy $421 \mathrm{~nm}$ transition," Optics Letters, vol. 34, no. 17, pp. 2548-2550, 2009.

[10] W. G. Jin, T. Wakui, T. Endo, H. Uematsu, T. Minowa, and H. Katsuragawa, "Specific mass shift in Gd I and Dy I," Journal of the Physical Society of Japan, vol. 70, no. 8, pp. 2316-2320, 2001.

[11] R. J. Lipert and S. C. Lee, "Isotope shifts and hyperfine structure of erbium, dysprosium, and gadolinium by atomicbeam diode-laser spectroscopy," Applied Physics B Photophysics and Laser Chemistry, vol. 57, no. 6, pp. 373-379, 1993.

[12] H. D. Kronfeldt, D. Ashkenasi, and H. M. Nikseresht, "Crossed-second-order effects in the isotope shift for ${ }^{170-166} \mathrm{Er}$ and ${ }^{168-166}$ Er in the configuration $4 f^{12} 6 s^{2}$," Zeitschrift für Physik D, vol. 22, no. 3, pp. 569-575, 1992.

[13] W. G. Jin, T. Horiguchi, M. Wakasugi, and Y. Yoshizawa, "Hyperfine structure and isotope shift in Er I by the atomicbeam laser spectroscopy," Journal of the Physical Society of Japan, vol. 59, no. 9, pp. 3148-3154, 1990.

[14] M. Wakasugi, T. Horiguchi, W. G. Jin, H. Sakata, and Y. Yoshizawa, "Changes of the nuclear charge distribution of Nd, Sm, Gd and Dy from optical Isotope shifts," Journal of the Physical Society of Japan, vol. 59, no. 8, pp. 2700-2713, 1990.

[15] A. Bernard, H. Brüggemeyer, and V. Pfeufer, "Changes in mean-square nuclear charge radii in Er from optical isotope shifts by laser-atomic-beam spectroscopy," Zeitschrift für Physik A, vol. 322, no. 1, pp. 1-11, 1985.

[16] V. Pfeufer, W. J. Childs, and L. S. Goodman, "J dependence of the isotope shift in the ground term of dysprosium I," Journal 
of the Optical Society of America B, vol. 1, no. 1, pp. 34-37, 1984.

[17] S. M. Afzal and S. A. Ahmad, "Isotope shift studies in the spectral lines of Dy I in the UV region: new assignments to $4 f^{9} 5$ d6s6p configuration," Spectrochimica Acta Part B: Atomic Spectroscopy, vol. 55, no. 1, pp. 97-102, 2000.

[18] D. Ashkenasi, S. Kröger, and H. D. Kronfeldt, "Finestructure, hyperfine structure and isotope shift of $4 f^{12} 6 s 7 s$ in Er I," European Physical Journal D, vol. 11, no. 2, pp. 197-205, 2000.

[19] W. G. Jin, Y. Nemoto, and T. Minowa, "J Dependence of isotope shifts at high-lying levels of Gd i," Journal of the Physical Society of Japan, vol. 78, no. 5, Article ID 055001, 2009.

[20] W. G. Jin, H. Nakai, M. Kawamura, and T. Minowa, "Highresolution ultraviolet laser spectroscopy in atomic erbium," Journal of the Physical Society of Japan, vol. 78, no. 1, Article ID 015001, 2009.

[21] W. G. Jin, Y. Nemoto, H. Nakai, M. Kawamura, and T. Minowa, "Isotope shifts in Gd I and Er I by UV laser spectroscopy," Journal of the Physical Society of Japan, vol. 77, no. 12, Article ID 124301, 2008.

[22] W. F. Meggers, C. H. Corliss, and B. F. Scribner, Tables of Spectral-Line Intensities, Part I - Arranged by Elements, NBS Monograph 145, US GPO, Washington, DC, USA, 1975.

[23] W. C. Martin, R. Zalubas, and L. Hagan, Atomic Energy Levels: The Rare-Earth Elements, NSRDS - NBS60, US GPO, Washington, DC, USA, 1978.

[24] J. J. McClelland, "Natural linewidth of the 401-nm lasercooling transition in Er I," Physical Review A, vol. 73, no. 6, Article ID 064502, 2006.

[25] K. Heilig and A. Steudel, "Changes in mean square nuclear charge radii from optical isotope shifts," Atomic Data and Nuclear Data Tables, vol. 14, no. 5-6, pp. 613-638, 1974.

[26] P. Aufmuth, K. Heilig, and A. Steudel, "Changes in meansquare nuclear charge radii from optical isotope shifts," Atomic Data and Nuclear Data Tables, vol. 37, no. 3, pp. 455-490, 1987.

[27] W. H. King, Isotope Shifts in Atomic Spectra, Plenum, New York, NY, USA, 1984. 


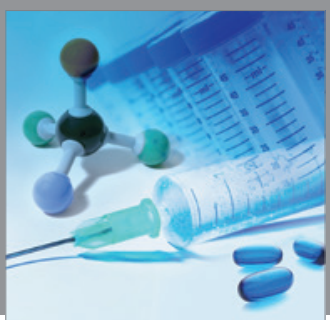

International Journal of

Medicinal Chemistry

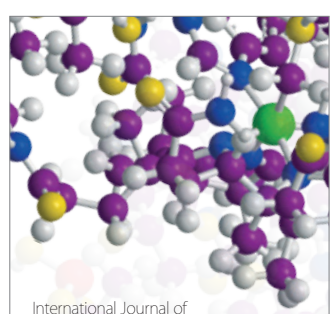

Carbohydrate Chemistry

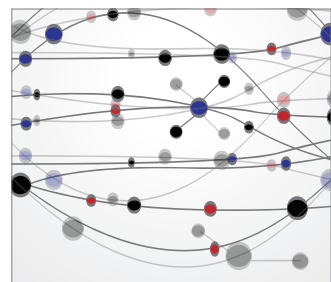

The Scientific World Journal
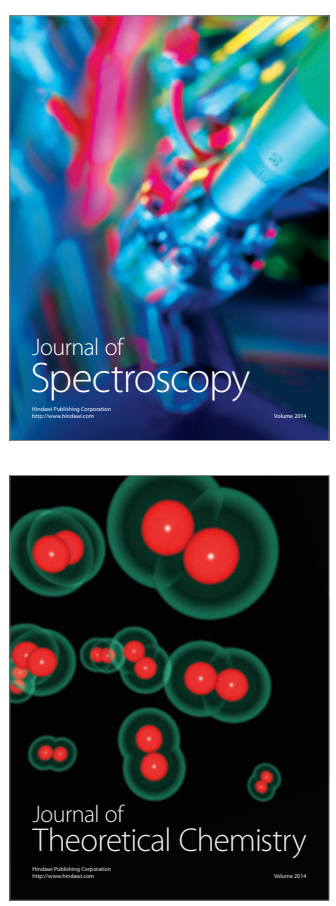
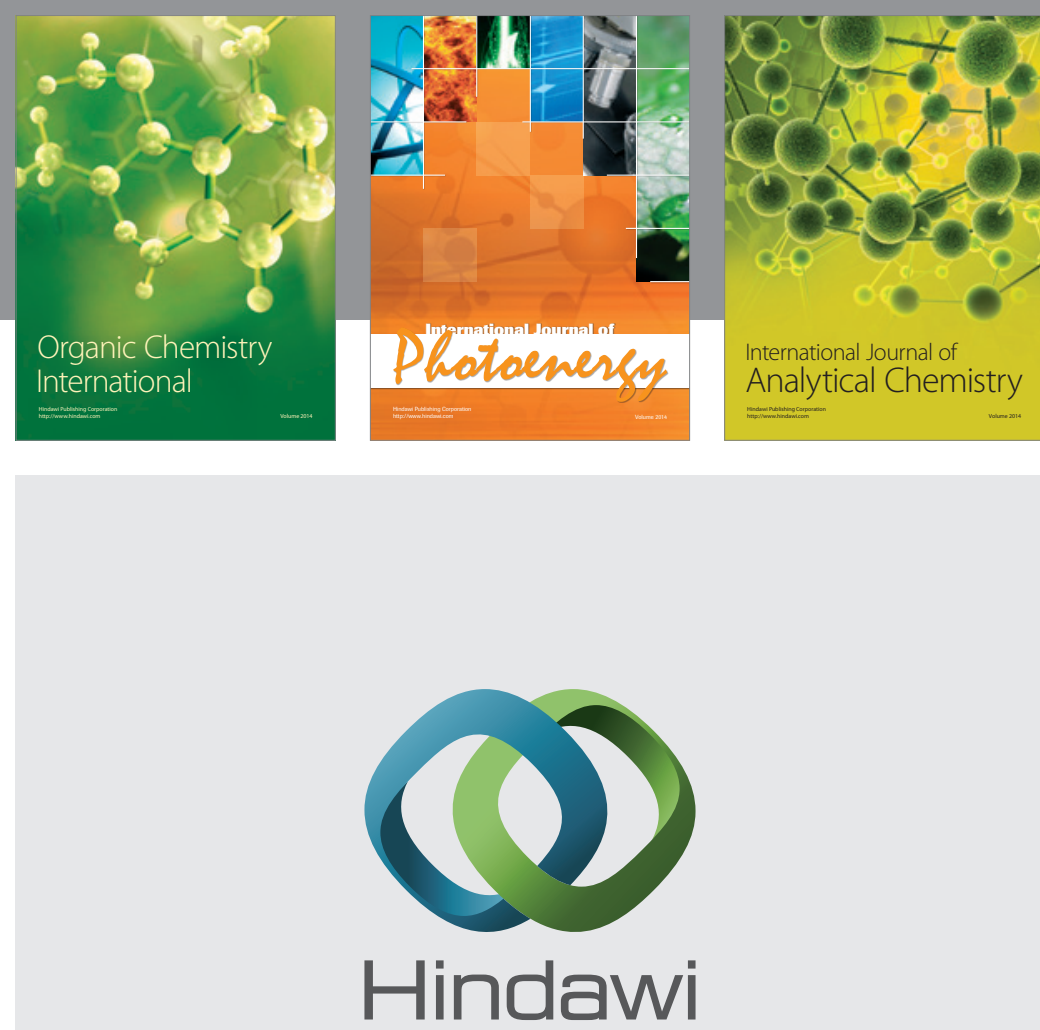

Submit your manuscripts at

http://www.hindawi.com
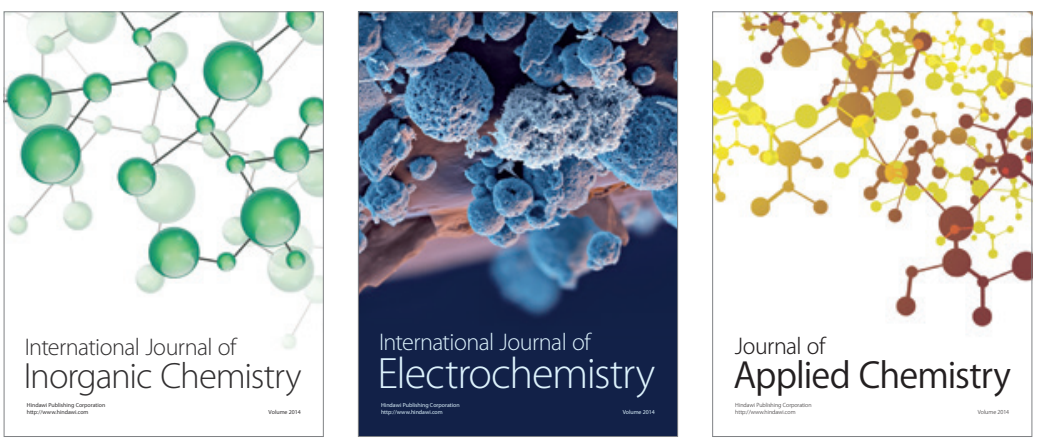

Journal of

Applied Chemistry
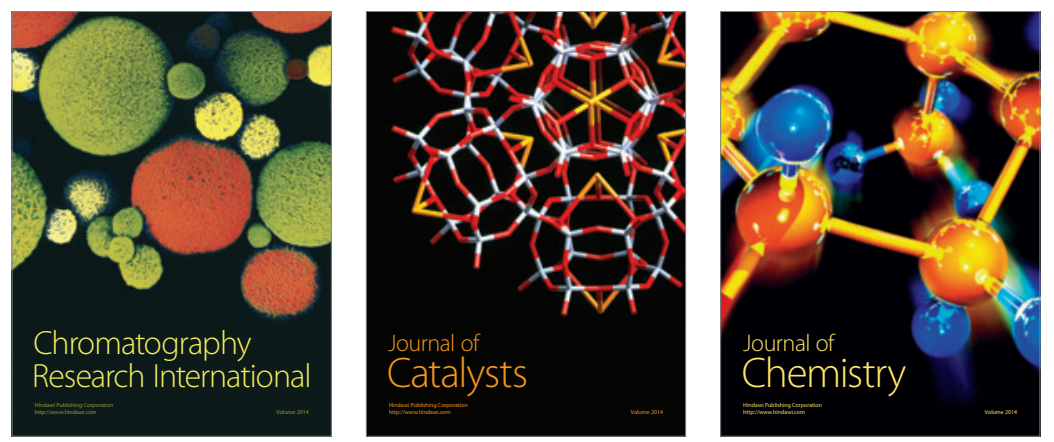
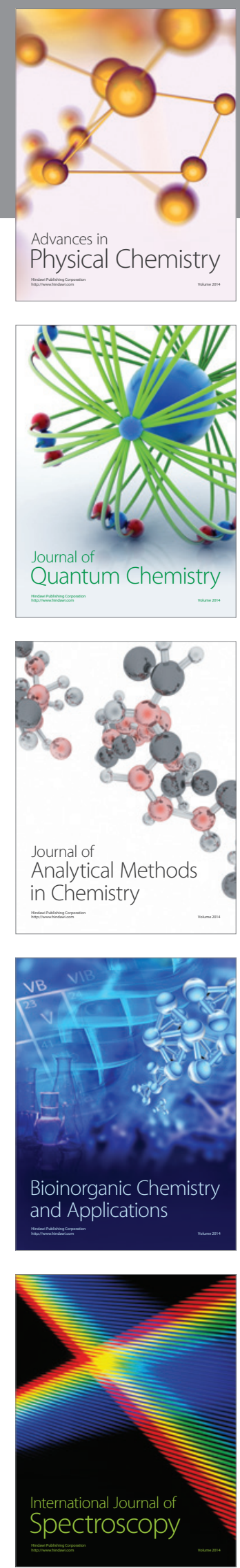